\title{
Selective depletion of cultured macrophages by magnetite nanoparticles modified with gelatin
}

\author{
YOSHIHIRO KOMOHARA ${ }^{1}$, RYUTA KAWAUCHI ${ }^{2}$, ERIKA MAKIYAMA ${ }^{2}$, KAZUKI MIKAMI $^{1}$, \\ HASITA HORLAD $^{1}$, YUKIO FUJIWARA ${ }^{1}$, TETSUYA KIDA ${ }^{3}$, MOTOHIRO TAKEYA $^{1}$ and TAKURO NIIDOME ${ }^{2}$ \\ ${ }^{1}$ Department of Cell Pathology, Graduate School of Medical Sciences, Kumamoto University, Kumamoto 860-8556; \\ ${ }^{2}$ Department of Applied Chemistry and Biochemistry, Graduate School of Science and Technology; \\ ${ }^{3}$ Magnesium Research Center, Kumamoto University, Kumamoto 860-8555, Japan
}

Received April 13,2016; Accepted May 5, 2017

DOI: $10.3892 / \mathrm{etm} .2017 .4640$

\begin{abstract}
Previous studies have indicated pro-tumor functions of macrophages in tumor progression in different types of malignant tumors. The detailed mechanisms of cell-cell interaction between macrophages and tumor cells have been investigated by means of in vitro co-culture experiments. The present study developed magnetite nanoparticles modified with gelatin that are specifically engulfed by macrophages and investigated methods to deplete these macrophages in co-culture experiments using a magnet. T98G glioma cell line and human monocyte-derived macrophages were mixed and co-cultured for 2 days. The T98G cells were isolated by depletion of the macrophages using the magnetite nanoparticles. mRNA expression of a number of pro-tumor molecules in the isolated T98G cells, with or without co-culture with macrophages, was then evaluated. The mRNA expression levels of chemokine (CC motif) ligand 2, interleukin-6 and macrophage-colony stimulating factor receptor (M-CSFR) were significantly upregulated in T98G cells by co-culture with macrophages $(\mathrm{P}<0.01)$. M-CSFR protein expression was also increased by co-culture with macrophages. The conditioned medium of co-cultured cells increased M-CSFR expression in T98G cells. Magnetite nanoparticles may be a novel tool not only for investigating the unique activation status of tumor cells in co-culture conditions, but also for targeting pro-tumor macrophages in tumor tissues.
\end{abstract}

\section{Introduction}

Recent advances have indicated that there is a notable involvement of macrophages in the progression of malignant tumors,

Correspondence to: Dr Yoshihiro Komohara, Department of Cell Pathology, Graduate School of Medical Sciences, Kumamoto University, Honjo 1-1-1, Kumamoto 860-8556, Japan

E-mail: ycomo@kumamoto-u.ac.jp

Key words: macrophage, tumor-associated macrophages, nanoparticle, glioma, macrophage-colony stimulating factor receptor including lymphoma and glioma, and macrophages are now of interest as target cells for therapy against malignant tumors $(1,2)$. Circulating monocytes infiltrate tumor tissues following their attraction via tumor cell-derived chemokines, such as chemokine (CC motif) ligand 2 [CCL2; also known as monocyte chemoattractant protein-1 (MCP)]. Activated macrophages are known to secrete a number of cytokines that are associated with cell growth, angiogenesis, matrix remodeling and immune suppression $(3,4)$. Macrophages that infiltrate into the tumor microenvironment are referred to as tumor-associated macrophages (TAMs). Since TAMs have pro-tumor functions in a number of malignant tumor types, TAMs are also considered as target cells for anti-tumor therapy. Previously, different materials, such as nanoparticles and nanocarriers, have been reported to improve anti-tumor therapy $(5,6)$.

TAM activation is induced by direct contact of the TAMs with tumor cells in the tumor microenvironment (1-4). Intracellular adhesion molecule-1 and membrane type macrophage-colony stimulating factor (M-CSF) are known to be involved in this direct cell-cell contact $(7,8)$. It has previously been demonstrated that the growth of a number of tumor cells, including glioma and lymphoma cells, was upregulated by direct co-culture with macrophages, and that signal transducer and activator of transcription (STAT) 3 activation was involved in this tumor cell activation $(9,10)$. However, the significance of cell-cell contact between macrophages and tumor cells remains unknown. Previous studies attempted to separate macrophages from tumor cells following direct co-culture experiments; however, it proved too difficult to separate the two types of cells $(8,11)$. Sorting methods using flow cytometry were not suitable for gene expression analysis because of RNA degradation during the procedure (11). Although anti-cluster of differentiation (CD) 14 or CD11b antibody-labeled magnet beads are commonly available to rapidly isolate monocytes/macrophages, downregulation of CD14/CD11b has been observed on activated macrophages when using these beads $(12,13)$. It is therefore necessary to develop novel and simple methods to separate macrophages from tumor cells following direct co-culture experiments.

The present study developed magnetite nanoparticles modified with gelatin that are specifically engulfed by macrophages, 
in addition to methods to deplete these macrophages in direct co-culture experiments by means of a magnet. By using these nanoparticles, the present study revealed that the expression of pro-tumor genes, including CCL2, interleukin (IL)-6, and M-CSF receptor (M-CSFR) were significantly upregulated in T98G glioma cells by direct co-culture with macrophages.

\section{Materials and methods}

Synthesis of magnetite nanoparticles. Fe(III) acetylacetonate $(1.09 \mathrm{~g}), 1,8$-octanediol $(0.09 \mathrm{~g}), 1$-octadecene $(21 \mathrm{ml})$, and oleylamine $(2.1 \mathrm{ml}$; all Wako Pure Chemical Industries, Ltd., Osaka, Japan) were loaded into a three-necked flask. The mixture was heated to $110^{\circ} \mathrm{C}$ for $30 \mathrm{~min}$ under vacuum. The temperature was then increased to $200^{\circ} \mathrm{C}$ under a $99.9 \%$ argon atmosphere and incubated for $2 \mathrm{~h}$ to produce magnetite nanoparticles. Following the reaction, the nanoparticles were further heated to $280^{\circ} \mathrm{C}$ and incubated for $1 \mathrm{~h}$ under an argon atmosphere. The resulting magnetite nanoparticles were washed 3 times by repeated precipitation from $100 \%$ hexane by adding excess $99 \%$ ethanol to remove any impurities from the magnetite surface. The synthesized magnetite nanoparticles were dispersed in hexane for storage.

Gelatin coating of magnetite nanoparticles. Following the removal of the hexane and dispersal of the magnetite nanoparticles by centrifugation at 1,200 x $\mathrm{g}$ for $30 \mathrm{~min}$, the magnetite nanoparticles were dried in vacuo. Gelatin $(0.13 \mathrm{~g}$; Nitta Gelatin Inc., Osaka, Japan) was added to $0.35 \mathrm{ml}$ distilled water and incubated for $2 \mathrm{~h}$ at room temperature. Following hydration, the gelatin was heated to $60^{\circ} \mathrm{C}$ and dissolved. The magnetite nanoparticles $(0.20 \mathrm{~g})$ were mixed with the gelatin solution at $60^{\circ} \mathrm{C}$ to produce a gel formation of the gelatin. This gel was dried following cooling, in vacuo, and the resultant black block was rubbed on an inkstone and gelatin-coated magnetite nanoparticles were obtained. The morphology of the gelatin-coated magnetite nanoparticles was observed using transmission electron microscopy (TEM; JEM-1400 plus; JEOL, Ltd., Tokyo, Japan). Samples were placed on carbon-coat copper grids (Okenshoji Co., Ltd., Tokyo, Japan), allowed to dry in the open air and then dried in vacuo. The size distribution of the magnetite nanoparticles was measured using dynamic light scattering analysis with the Zetasizer Nano ZS ${ }^{\text {тм }}$ (Malvern Instruments, Ltd. Malvern, UK).

Macrophages. Peripheral blood mononuclear cells were obtained from healthy volunteer donors (six male healthy donors recruited from Kumamoto University; Kumamoto, Japan; 25-40 years old) recruited between April 2015 and February 2016 whom had all provided written informed consent for the use of their cells in accordance with the study protocols approved by the Kumamoto University Hospital Review Board (no. G309; Kumamoto, Japan). CD14+ monocytes were isolated using CD14-microbeads (Miltenyi Biotec GmbH, Bergisch Gladbach, Germany), and the cells were then cultured in $2 \%$ human serum, $1 \mathrm{ng} / \mathrm{ml}$ granulocyte macrophage-colony stimulating factor (GM-CSF) and $50 \mathrm{ng} / \mathrm{ml} \mathrm{M-CSF} \mathrm{(all} \mathrm{Wako} \mathrm{Pure}$ Chemical Industries, Ltd., Osaka, Japan) for 7 days at $37^{\circ} \mathrm{C}$ to induce macrophages.
Glioma cell line. The human glioma cell line, T98G, was purchased from the American Type Culture Collection (Manassas, VA, USA), and was maintained in Dulbecco's modified Eagle medium (DMEM)/Ham's F-12 supplemented with $10 \%$ fetal bovine serum (FBS; all Wako Pure Chemical Industries, Ltd.). T98G cells were infected with a lentivirus encoding green fluorescent protein (GFP; Santa Cruz Biotechnology, Inc., Dallas, TX, USA) and T98G GFP-expressing (T98G-GFP) cells were selected using puromycin, as described in the manufacturer's protocol (Santa Cruz Biotechnology, Inc.).

Uptake of magnetite nanoparticles by human macrophages and T98G cells. The gelatin-coated magnetite nanoparticles $(10 \mu \mathrm{l} ; 7.3 \mathrm{mg} / \mathrm{ml})$ were added to human macrophages obtained from healthy volunteers and to the human glioblastoma T98G cells, which were then cultured for up to $2 \mathrm{~h}$ at $37^{\circ} \mathrm{C}$ in $100 \mu \mathrm{l}$ DMEM/Ham's F-12 supplemented with 10\% FBS in LabTech Chamber Slides (Thermo Fisher Scientific, Inc., Waltham, MA, USA). Uptake of the nanoparticles was observed using an optical microscope (BX51; Olympus Corp., Tokyo, Japan) following hematoxylin staining for $1 \mathrm{~min}$ at room temperature.

Separation of human macrophages from the co-culture of macrophages and T98G cells. Human macrophages and T98G cells $\left(2 \times 10^{5}\right.$ and $1 \times 10^{5}$ cells/well in a 6 -well culture plate, respectively) were mixed and co-cultured at $37^{\circ} \mathrm{C}$ under $5 \% \mathrm{CO}_{2}$. After 2 days, the magnetite nanoparticles were added to the culture medium. Following a $1-\mathrm{h}$ incubation at $37^{\circ} \mathrm{C}$, the cells were collected into a centrifuge tube using cell dissociating buffer (Thermo Fisher Scientific, Inc.). Cells that had taken up the magnetite nanoparticles were collected using a magnet (MPC-S, DYNAL ${ }^{\circledR}$; Invitrogen; Thermo Fisher Scientific, Inc.). All cells, magnet-collected or not, were attached to glass slides using Cytospin (800 x g for $5 \mathrm{~min}$ ar room temperatyre; Thermo Fisher Scientific, Inc.).

Immunostaining. All procedures were performed at $37^{\circ} \mathrm{C}$. Cells were fixed with cold acetone (100\%) and subsequently incubated with $1 \%$ FBS in PBS for blocking (10 min) and then anti-CD204 antibody (1:100; clone no. SRA-E5; MAB1710, Cosmo Bio, Tokyo, Japan) for $1 \mathrm{~h}$ in order to detect TAMs. Following this, cells were incubated with secondary antibody (1:2; 414131; Nichirei Biosciences, Inc., Tokyo, Japan) for $30 \mathrm{~min}$. Reactions were visualized under light microscope using a diaminobenzidine (DAB) substrate kit as described in manufacture's protocol (Nichirei Biosciences, Inc.).

Magnet beads. Anti-human CD14 antibody-labeled microbeads and a magnetic column were purchased from Miltenyi Biotec $\mathrm{GmbH}$ were used, according to the manufacturer's protocol.

Reverse transcription-quantitative polymerase chain reaction (RT-qPCR). The mRNA expression in T98G cells was evaluated using RT-qPCR, as previously described (14). Total RNA was extracted with RNAiso Plus (Takara Bio, Inc., Otsu, Japan), following the manufacturers protocol. RNA was reverse transcribed using a PrimeScript RT Reagent kit, according to the manufacturer's instructions, and DNase from Takara Bio, 
Table I. Primer sequences for reverse transcription-quantitative polymerase chain reaction.

\begin{tabular}{lll}
\hline Gene & Direction & Sequence $\left(5^{\prime}-3^{\prime}\right)$ \\
\hline Chemokine (CC motif) ligand 2 & $\mathrm{~F}$ & CATAGCAGCCACCTTCATTCC \\
Interleukin-6 & $\mathrm{R}$ & TGCACTGAGATCTTCCTATTGGTG \\
& $\mathrm{F}$ & ATGTGTGAAAGCAGCAAAGAGG \\
$\mathrm{O}(6)$-methylguanine-DNA methyltansferase & $\mathrm{R}$ & GTGATGATTTTCACCAGGCAAG \\
& $\mathrm{F}$ & GGCCGAAACTGAGTATGTGC \\
Transforming growth factor- $\beta$ & $\mathrm{R}$ & CCTTTAATACAGCGGTGCCT \\
& $\mathrm{F}$ & AGCAACAATTCCTGGCGATAC \\
Vascular endothelial growth factor-A & $\mathrm{R}$ & GCGAAAGCCCTCAATTTCC \\
$\beta$-actin & $\mathrm{F}$ & CAGGAGTACCCTGATGAGATCG \\
& $\mathrm{R}$ & TCTGCATGGTGATGTTGGAC \\
\hline
\end{tabular}

F, forward; R, reverse.

Inc. The complementary DNA product $(25 \mu \mathrm{l})$ was amplified as follows: $94^{\circ} \mathrm{C}$ for $5 \mathrm{~min}$ followed by 40 cycles of $94^{\circ} \mathrm{C}$ for $30 \mathrm{sec}$ and $60^{\circ} \mathrm{C}$ for $30 \mathrm{sec}$. qPCR was performed using TaqMan polymerase with SYBR-Green fluorescence (Takara Bio, Inc.) with an ABI PRISM ${ }^{\circledR} 7300$ Sequence Detection System (Applied Biosystems; Thermo Fisher Scientific, Inc.). The sequences of the primers were designed using the Primer3 website (bioinfo.ut.ee/primer3-0.4.0/) and were synthesized by Hokkaido System Science Co., Ltd., (Tokyo, Japan). The primer sequences are presented in Table I. The internal control gene used was $\beta$-actin, and three parallel wells were set up for each DNA sample ( $25 \mu \mathrm{l} /$ well). The relative expression level was determined using the $2^{-\Delta \Delta \mathrm{Cq}}$ normalization method (15).

Flow cytometry. Cells were detached using cell dissociation buffer (Thermo Fisher Scientific, Inc.) and incubated with blocking solution (422302; BioLegend, San Diego, CA, USA) for $10 \mathrm{~min}$ at room temperature. Cells were then stained using anti-M-CSFR antibody $(1: 1,000$; rabbit polyclonal; LS-C167079; LifeSpan Bioscience, Inc., Seattle, WA, USA) or control rabbit immunoglobulin (Ig)G (AB-105C; Santa Cruz Biotechnology, Inc.) with Fc receptor blocking solution (BioLegend, Inc.). The cells were then incubated for $30 \mathrm{~min}$ at room temperature with allophycocianin (APC)-labeled anti-rabbit antibody (1:1; 406414; BioLegend, Inc.), and staining signals were evaluated using FACSverse ${ }^{\mathrm{TM}}$ (BD Biosciences, Franklin Lakes, NJ, USA) and FACSuite software 21 (BD Biosciences).

Western blot analysis. Cells were lysed in ice-cold lysis buffer [50 mM Tris (pH 8.0), $1 \mathrm{mM}$ EDTA, $150 \mathrm{mM} \mathrm{NaCl}$ and $1 \%$ NP-40] and a protease inhibitor cocktail (Sigma-Aldrich; Merck KGaA, Darmstadt, Germany). Following electrophoresis of $10 \mu \mathrm{g} /$ lane protein using $10 \%$ SDS-PAGE, the polyvinylidene difluoride membrane was incubated for $1 \mathrm{~h}$ at room temperature with anti-M-CSFR antibody $(1: 1,000$; rabbit polyclonal; 3152; Cell Signaling Technology, Inc., Danvers, MA, USA) or anti- $\beta$-actin antibody $(1: 2,000$; mouse monoclonal; sc47778; Santa Cruz Biotechnology, Inc.) Horseradish peroxidase-goat anti-mouse or rabbit IgG (1:1,000; 31430; 31460; Thermo Fisher Scientific, Inc.) were used as the secondary antibody and incubated for $30 \mathrm{~min}$ at room temperature. Immunoreactive bands were visualized using the Pierce Western Blotting Substrate Plus kit (Pierce; Thermo Fisher Scientific, Inc.) and ImageQuant ${ }^{\mathrm{TM}}$ LAS-4000 mini (GE Healthcare Life Sciences, Uppsala, Sweden).

Statistical analysis. Statistical analysis of PCR data was completed using Student's t-tests using EXEL 2016 software (Redmond, WA, USA). Data are presented as the mean \pm standard deviation of triplicate results. $\mathrm{P}<0.05$ was considered to indicate a statistically significant difference.

\section{Results}

Characterization of magnetite nanoparticles. Magnetite nanoparticles were prepared by reducing Fe(III) in the presence of 1,8-octanediol, 1-octadecene and oleylamine by heating under an argon atmosphere, followed by the use of previously reported methods (16). The magnetite nanoparticles were mixed with a gelatin solution at $60^{\circ} \mathrm{C}$ and, following cooling, a gelatin gel containing the magnetite nanoparticles was prepared. The gel was dried in vacuo and the solid block was rubbed on an inkstone with water. This method is the same as the traditional method used to prepare Chinese gelatin-coated carbon black ink. Nanoparticles of $\sim 20 \mathrm{~nm}$ in diameter were observed under TEM and the particles formed aggregates (Fig. 1). Dynamic light scattering analysis indicated that the mean diameter of the aggregates was $800 \mathrm{~nm}$. The TEM image indicated a shadow on the surface of these particles, confirming that the magnetite nanoparticles were coated with a gelatin coating that was $\sim 4 \mathrm{~nm}$ thick (Fig. 1).

Uptake of magnetite by macrophages and T98G cells. The gelatin-coated magnetite nanoparticles were added to human macrophages and glioblastoma T98G cells. Uptake of the 

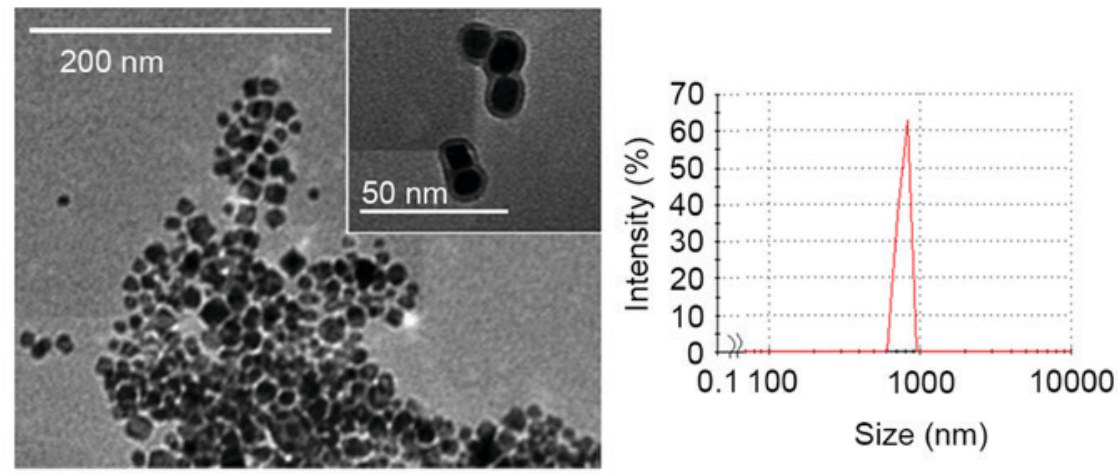

Figure 1. Transmission electron microscopy image (magnification, x10,000) of gelatin-coated magnetite nanoparticles and the size distribution of the gelatin magnetite nanoparticles.
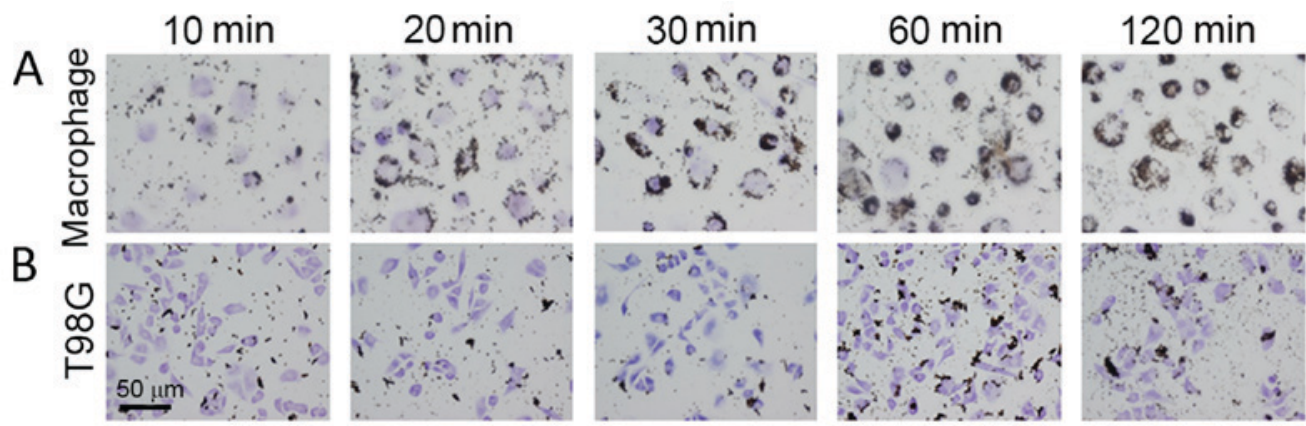

Figure 2. Hematoxylin staining of cells following the addition of gelatin-coated magnetite nanoparticles for 10, 20, 30, 60 or 120 min to (A) cultured macrophages or (B) T98G cells. Magnification, x200. Scale bar, $50 \mu \mathrm{m}$.

nanoparticles by macrophage cells was observed at $10 \mathrm{~min}$, and the amount of nanoparticles taken up increased with the incubation time (Fig. 2A). Since the nanoparticles were observed as dots, it is possible that they were internalized by an endocytic pathway. T98G cells also internalized the nanoparticles; however, the amount internalized was lower than that in the human macrophages (Fig. 2B).

Separation of human macrophages from co-culture of macrophages and T98G cells. In order to determine whether the magnetite nanoparticles may be used to separate macrophages from co-cultures with $\mathrm{T} 98 \mathrm{G}$ cells, the nanoparticles were added to a mixture of these cells. Once the nanoparticles were taken up by the cells, any cells with magnetite nanoparticles were collected using a magnet and analyzed (Fig. 3A). CD204 is a specific marker of macrophages and, based on the expression of this marker, $\sim 30 \%$ of the co-cultured cells were macrophages (Fig. 3B). As presented in Fig. 3C, the majority of the collected cells were stained with CD204, indicating that they were macrophages. However, certain CD204-negative T98G cells were also detected in the collected cells, and the percentage of contaminating T98G cells was $>5 \%$. By contrast, the vast majority of the cells not collected with the magnet were not stained with CD204, and contamination with CD204-positive macrophages was $<1 \%$ (Fig. 3C). These results indicated that, while the purity of the collected macrophages was not high, the purity of the T98G cells in the uncollected fraction was high enough that the cells could be used for further analysis. In addition, the results obtained using magnetite nanoparticles were compared with those obtained using commercially available anti-CD14 antibody-labeled microbeads and a magnet column. The purity of the separated tumor cells using commercially available magnet beads was $12-14 \%$, which was higher than that obtained using magnetite nanoparticles (Fig. 3D).

Enhanced expression of CCL2, IL-6 and M-CSFR by T98G cells co-cultured with macrophages. It has been previously demonstrated that co-culture with macrophages induces activation of T98G cells; however, the gene expression profile of T98G cells was not evaluated (8). Therefore, in the present study, the gene expression of CCL2, IL-6, $\mathrm{O}(6)$-methylguanine-DNA methyltansferase (MGMT), transforming growth factor- $\beta$ (TGF- $\beta$ ), vascular endothelial growth factor-A (VEGF-A) and M-CSFR in control T98G cells and in the T98G cells isolated from a co-culture with macrophages was investigated by RT-qPCR. Upregulation of CCL2, IL-6, and M-CSFR mRNA expression was demonstrated in T98G cells that had been co-cultured with macrophages compared with control cells ( $\mathrm{P}<0.01$; Fig. 4A). No significant differences in the mRNA expression levels of MGMT, TGF- $\beta$ or VEGF-A were observed (data not shown). M-CSFR expression in the cells was further evaluated by flow cytometry to confirm the increased expression of M-CSFR in T98G cells co-cultured with macrophages compared with control cells. In this experiment, T98G-GFP cells and macrophages were directly mixed and co-cultured for 2 days, following which the cells were stained with an anti-M-CSFR antibody. As demonstrated in Fig. 4B, overexpression of 
A

T98G

Macrophage

\section{Gelatin-coated magnetite} nanoparticle
Isolated tumor cells

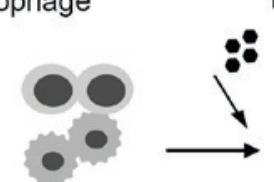

Co-culture

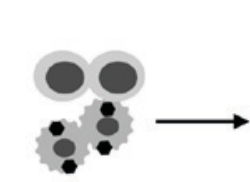

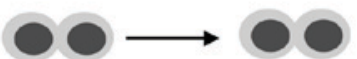

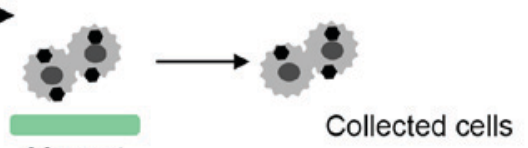

Magnet

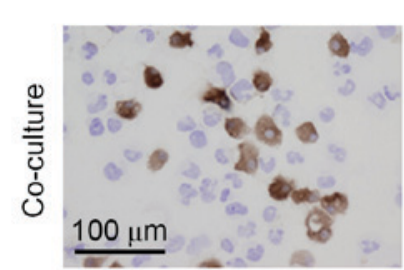

C
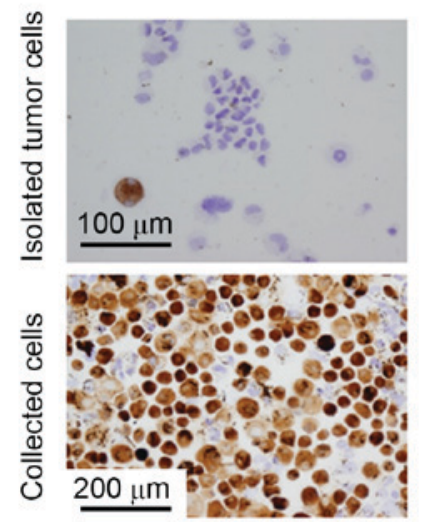

D

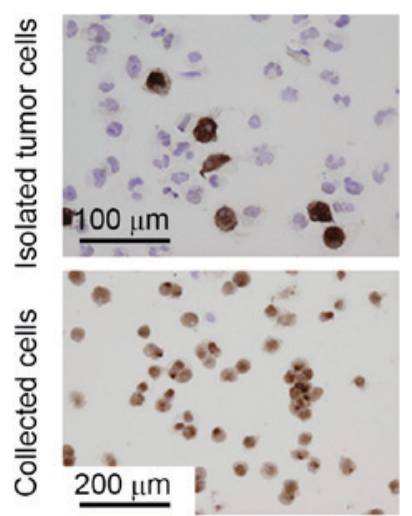

Figure 3. Immunostaining of the macrophage marker CD204 in magnet-collected and uncollected cells. (A) Schematic of cell separation using gelatin-coated magnetite nanoparticles. Cells were mixed and co-cultured for 2 days, following which magnetite nanoparticles were added for 30 min. The cells were then detached using cell dissociation buffer and separated using a magnet. (B) Co-cultured cells were attached to a glass slide and were immunostained for CD204. Cells were then separated using (C) gelatin-coated magnetite nanoparticles or (D) commercially available anti-CD14 antibody-labeled microbeads, attached to a glass slide and immunostained for CD204. The data are representative of two or three independent experiments. CD, cluster of differentiation.

A
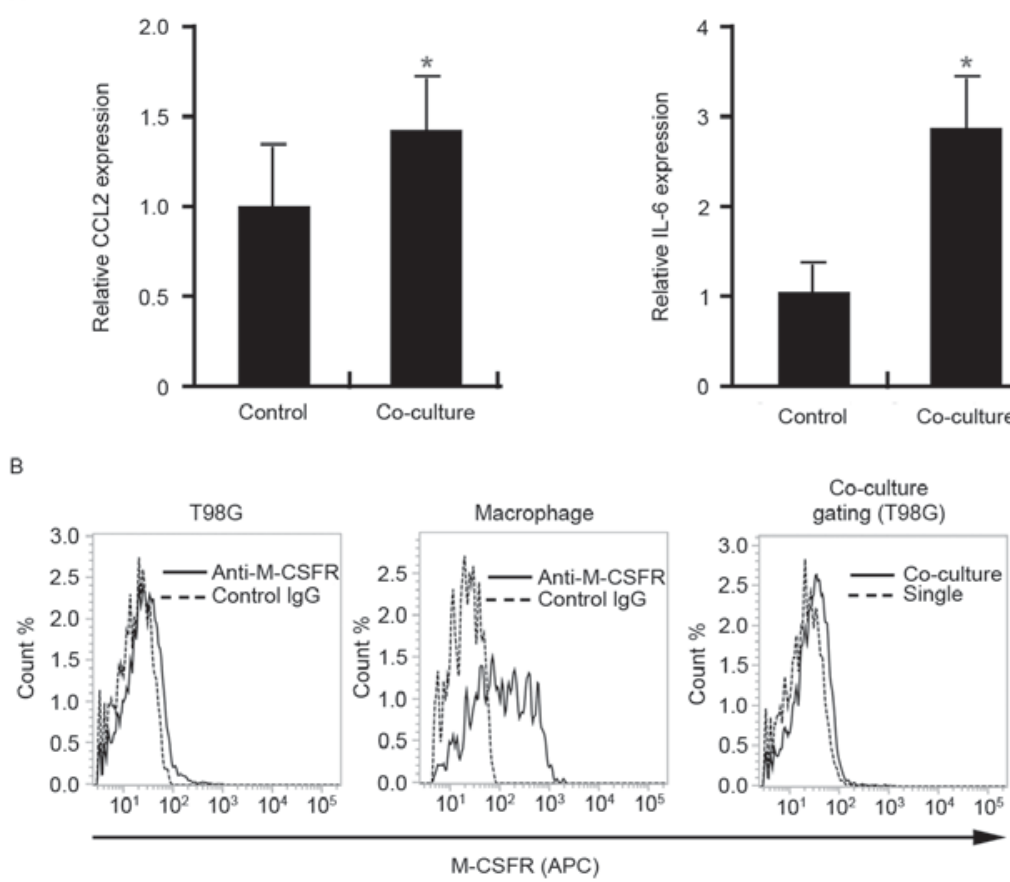
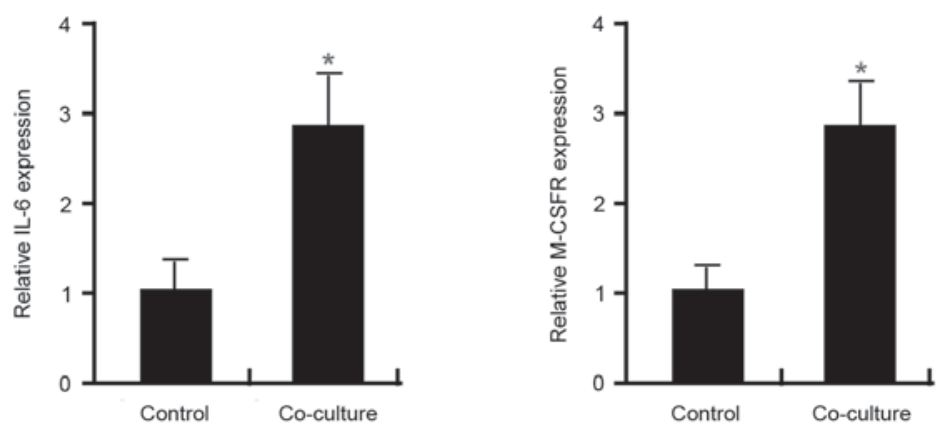

C

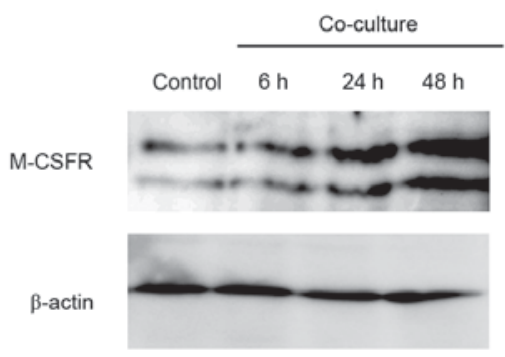

Figure 4. Expression of pro-tumor molecules by T98G cells following co-culture with macrophages. (A) mRNA expression of CCL2, IL-6 and M-CSFR, normalized to $\beta$-actin, in control T98G cells and T98G cells following their isolation from co-culture with macrophages by depletion of the macrophages using gelatin-coated magnetite nanoparticles. (B) M-CSFR expression on the cell surface of T98G-GFP cells and macrophages was analyzed using flow cytometry. M-CSFR expression on macrophages and T98G-GFP cells was distinguished by gating the green fluorescence of GFP. (C) T98G cells were cultured with the conditioned medium of a co-culture of T98G cells and macrophages, following which M-CSFR expression was evaluated by western blot analysis. $\beta$-actin expression was analyzed as a loading control. The data are representative of at least two or three independent experiments. ${ }^{*}<0.01$ vs. the control. CCL2, chemokine (CC motif) ligand 2; IL-6, interleukin-6; M-CSFR, macrophage-colony stimulating factor receptor; GFP, green fluorescence protein; IgG, immunoglobulin G; APC, allophycocyanin. 
M-CSFR was observed on T98G-GFP cells when they were co-cultured with macrophages. In addition to analysis using flow cytometry, the effect of the conditioned medium of a co-culture of T98G cells and macrophages on M-CSFR expression by $\mathrm{T} 98 \mathrm{G}$ cells was evaluated using western blot analysis. T98G cells were cultured with the conditioned medium of co-cultured (T98G and macrophages) cells for 6 , 24 , or $48 \mathrm{~h}$ following which cell lysates were analyzed by western blot analysis. M-CSFR expression in T98G cells was markedly increased in a time dependent manner by stimulation of the cells with the conditioned medium compared with control cells (Fig. 4C).

\section{Discussion}

The present study developed a simple and easy method for separating macrophages from tumor cells in a co-culture system. The purity of the tumor cells was $<90 \%$ following a previous attempt to separate macrophages from tumor cells in a co-culture system using commercially available anti-CD14 microbeads and a magnet column (unpublished data). Downregulation of the CD14 antigen in macrophages is considered to be the reason for this result. As the cost of preparing magnetite nanoparticles is markedly lower than that of commercially available antibody-labeled microbeads, the methods presented in the present study may be more acceptable or more convenient than such microbeads for separating macrophages from tumor cells in a co-culture system. Although the purity of the obtained tumor cells is sufficient for further experiments aimed at evaluating mRNA expression, the purity of the obtained macrophages was affected by tumor cell contamination. Tumor cell contamination was considered to be due to non-specific binding of magnetite nanoparticles to tumor cells, and further studies are necessary to reduce the non-specific binding of magnetite nanoparticles by modulating the coating materials or methods.

In the present study, magnetite nanoparticles were coated with gelatin because of its low cost and specific binding affinity to macrophages (17). Nanoparticles or nanocarriers targeting macrophages have been developed and have been demonstrated to be useful for anti-tumor therapy targeting TAMs $(18,19)$. The present study initially aimed to develop the magnetite nanoparticles used in the present study for use as nanoparticles for targeting TAMs. These magnetite nanoparticles were assessed previously by the authors of the present study in a murine tumor implantation model. However, magnetite nanoparticles that were injected into a vein mainly localized to the liver, spleen and lung, and not to tumor tissues (unpublished data). Therefore, it may be necessary to develop additional methods to specifically deliver the magnetite nanoparticles to tumor tissues in vivo.

In the present study, pro-tumor molecules including, CCL2, IL-6 and M-CSFR were significantly upregulated in T98G cells following co-culture with macrophages. CCL2 is also known as MCP-1 and was first identified in glioblastoma cell lines (20). CCL2 was then identified to be expressed by different types of tumor cells (20). CCL2 is closely involved in the chemotaxis of TAMs in the tumor microenvironment (20). TAMs are believed to accelerate tumor progression and development, therefore CCL2/CC receptor type 2 binding is considered a promising target for additional anti-tumor therapy (21). IL-6 has a well-known association with tumor cell proliferation, survival and tumorigenesis via activation of Janus kinases and STATs (22). IL-6 secreted by mesenchymal cells and tumor cells activates the STAT3 signal, which is involved in the maintenance and tumorigenicity of glioma stem cells in glioma (23). Therefore, STAT3 is considered to be a therapeutic target for glioma. M-CSFR, also known as c-fms, is not only a well-known oncogene that is involved in tumor proliferation and survival, but is expressed in macrophages and is associated with macrophage activation (24). M-CSFR inhibition abrogates glioma progression by inhibiting the pro-tumor activation of TAMs (25). Although there have been a number of research studies regarding M-CSF production from glioma cells (26-28), to the best of our knowledge, there is no published research assessing M-CSFR expression in glioma cells, other than a previous study that reported the activation of M-CSFR in both glioma cells and TAMs in human glioma samples (8). The observations indicated that M-CSFR expression in cultured glioma cell lines is markedly lower than that in macrophages, and may suggest that M-CSFR expression in glioma cells may not be of particular interest for further research. However, the present study demonstrated that M-CSFR expression in T98G glioma cells was markedly upregulated by cell-cell interaction with TAMs. M-CSFR is known to be closely involved in tumor progression in other malignant tumors (29) and therefore, the function of the observed M-CSFR activation in glioma cells may be potentially associated with glioma progression.

In conclusion, the present study developed gelatin-coated magnetite nanoparticles for use in separating macrophages from tumor cells in a co-culture system, and demonstrated that certain pro-tumor molecules are induced in glioma cells by cell-cell interaction with macrophages. Although the methods used to coat the magnetite nanoparticles require improvement in future studies to inhibit their non-specific binding to tumor cells, magnetite nanoparticles are cheaper and easier to handle than antibody-labeled microbeads. Magnetite nanoparticles may be a novel tool not only for targeting TAMs, but also for investigation of the unique activation status of tumor cells in co-culture conditions.

\section{Acknowledgements}

The authors of the present study would like to thank Ms. Emi Kiyota, Mr. Osamu Nakamura, Ms. Yui Hayashida and Mr. Takenobu Nakagawa (Department of Cell Pathology, Kumamoto University, Kumamoto, Japan) for their technical assistance. The present study was supported by a grant from the Ministry of Education, Culture, Sports, Science and Technology of Japan (grant no. 25460497).

\section{References}

1. Casey SC, Amedei A, Aquilano K, Azmi AS, Benencia F, Bhakta D, Bilsland AE, Boosani CS, Chen S, Ciriolo MR, et al: Cancer prevention and therapy through the modulation of the tumor microenvironment. Semin Cancer Biol 35 (Suppl): S199-S223, 2015.

2. Komohara Y, Fujiwara Y, Ohnishi $\mathrm{K}$ and Takeya $\mathrm{M}$ : Tumor-associated macrophages: Potential therapeutic targets for anti-cancer therapy. Adv Drug Deliv Rev 99: 180-185, 2016. 
3. Pollard JW: Tumour-educated macrophages promote tumour progression and metastasis. Nat Rev Cancer 4: 71-78, 2004.

4. Kitamura T, Qian BZ and Pollard JW: Immune cell promotion of metastasis. Nat Rev Immunol 15: 73-86, 2015.

5. Naguib YW and Cui Z: Nanomedicine: The promise and challenges in cancer chemotherapy. Adv Exp Med Biol 811: 207-233, 2014.

6. Amoozgar Z and Goldberg MS: Targeting myeloid cells using nanoparticles to improve cancer immunotherapy. Adv Drug Deliv Rev 91: 38-51, 2015.

7. Komohara Y, Jinushi M and Takeya M: Clinical significance of macrophage heterogeneity in human malignant tumors. Cancer Sci 105: 1-8, 2014.

8. Komohara Y, Horlad H, Ohnishi K, Fujiwara Y, Bai B, Nakagawa T, Suzu S, Nakamura H, Kuratsu J and Takeya M. Importance of direct macrophage-tumor cell interaction on progression of human glioma. Cancer Sci 103: 2165-2172, 2012.

9. Usami Y, Ishida K, Sato S, Kishino M, Kiryu M, Ogawa Y, Okura M, Fukuda Y and Toyosawa S: Intercellular adhesion molecule-1 (ICAM-1) expression correlates with oral cancer progression and induces macrophage/cancer cell adhesion. Int J Cancer 133: 568-578, 2013.

10. Komohara Y, Niino D, Ohnishi K, Ohshima K and Takeya M Role of tumor-associated macrophages in hematological malignancies. Pathol Int 65: 170-176, 2015.

11. Russell JN, Clements JE and Gama L: Quantitation of gene expression in formaldehyde-fixed and fluorescence-activated sorted cells. PLoS One 8: e73849, 2013.

12. Ruppert J, Schütt C, Ostermeier D and Peters JH: Down-regulation and release of CD14 on human monocytes by IL-4 depends on the presence of serum or GM-CSF. Adv Exp Med Biol 329: 281-286, 1993

13. Prieto J, Eklund A and Patarroyo M: Regulated expression of integrins and other adhesion molecules during differentiation of monocytes into macrophages. Cell Immunol 156: 191-211, 1994.

14. Motoshima T, Komohara Y, Horlad H, Tsukamoto H, Fujita M, Saito Y, Tanoue K, Kasejima Y, Sugiyama Y, Kawano Y, et al: CXCL10 and CCL2 mRNA expression in monocytes is inversely correlated with the HLA-DR lower fraction of monocytes in patients with renal cell carcinoma. Oncol Lett 11: 1911-1916, 2016.

15. Livak KJ and Schmittgen TD: Analysis of relative gene expression data using real-time quantitative PCR and the 2(-Delta Delta C(T)) Method. Methods 25: 402-408, 2001.

16. Sun S and Zeng H: Size-controlled synthesis of magnetite nanoparticles. J Am Chem Soc 124: 8204-8205, 2002.

17. Gudewicz PW, Molnar J, Lai MZ, Beezhold DW, Siefring GE Jr, Credo RB and Lorand L: Fibronectin-mediated uptake of gelatin-coated latex particles by peritoneal macrophages. J Cell Biol 87: 427-433, 1980
18. Niu M, Naguib YW, Aldayel AM, Shi YC, Hursting SD, Hersh MA and Cui Z: Biodistribution and in vivo activities of tumor-associated macrophage-targeting nanoparticles incorporated with doxorubicin. Mol Pharm 11: 4425-4436, 2014.

19. Zhu S, Niu M, O'Mary H and Cui Z: Targeting of tumor-associated macrophages made possible by PEG-sheddable, mannose-modified nanoparticles. Mol Pharm 10: 3525-3530, 2013.

20. Yoshimura T, Robinson EA, Tanaka S, Appella E, Kuratsu J and Leonard EJ: Purification and amino acid analysis of two human glioma-derived monocyte chemoattractants. J Exp Med 169: 1449-1459, 1989

21. Qian BZ, Li J, Zhang H, Kitamura T, Zhang J, Campion LR, Kaiser EA, Snyder LA and Pollard JW: CCL2 recruits inflammatory monocytes to facilitate breast-tumour metastasis. Nature 475: 222-225, 2011.

22. Fisher DT, Appenheimer MM and Evans SS: The two faces of IL-6 in the tumor microenvironment. Semin Immunol 26: 38-47, 2014.

23. Hossain A, Gumin J, Gao F, Figueroa J, Shinojima N, Takezaki T, Priebe W, Villarreal D, Kang SG, Joyce C, et al: Mesenchymal stem cells isolated from human gliomas increase proliferation and maintain stemness of glioma stem cells through the IL-6/gp130/STAT3 pathway. Stem Cells 33: 2400-2415, 2015.

24. Hamilton JA: Colony-stimulating factors in inflammation and autoimmunity. Nat Rev Immunol 8: 533-544, 2008.

25. Pyonteck SM, Akkari L, Schuhmacher AJ, Bowman RL, Sevenich L, Quail DF, Olson OC, Quick ML, Huse JT, Teijeiro V, et al: CSF-1R inhibition alters macrophage polarization and blocks glioma progression. Nat Med 19: 1264-1272, 2013.

26. De I, Steffen MD, Clark PA, Patros CJ, Sokn E, Bishop SM, Litscher S, Maklakova VI, Kuo JS, Rodriguez FJ and Collier LS: CSF1 overexpression promotes high-grade glioma formation without impacting the polarization status of glioma-associated microglia and macrophages. Cancer Res 76: 2552-2560, 2016.

27. Sielska M, Przanowski P, Wylot B, Gabrusiewicz K, Maleszewska M, Kijewska M, Zawadzka M, Kucharska J, Vinnakota K, Kettenmann H, et al: Distinct roles of CSF family cytokines in macrophage infiltration and activation in glioma progression and injury response. J Pathol 230: 310-321, 2013.

28. Bender AM, Collier LS, Rodriguez FJ, Tieu C, Larson JD, Halder C, Mahlum E, Kollmeyer TM, Akagi K, Sarkar G, et al: Sleeping beauty-mediated somatic mutagenesis implicates CSF1 in the formation of high-grade astrocytomas. Cancer Res 70: 3557-3565, 2010

29. Patsialou A, Wyckoff J, Wang Y, Goswami S, Stanley ER and Condeelis JS: Invasion of human breast cancer cells in vivo requires both paracrine and autocrine loops involving the colony-stimulating factor-1 receptor. Cancer Res 69: 9498-9506, 2009. 